\title{
3D Seismic Survey Technique in Less Explored Region
}

\author{
Tang Hongwei* and He Wenxin
}

\author{
CCTEG Xi'an Research Institute, Xi'an City, Shaaxi Province, 710077, P.R. China
}

\begin{abstract}
By summarizing the experiences of routine acquisition geometry designs, and using the 3D seismic survey data of a less explored region for instance, this paper put forward an acquisition geometry design method for less explored region plus the geological stratum calibration by reflection wave and time-depth conversion method. After drilling data and roadway data verification, the re-argumentation of acquisition geometry parameters for parameter determination and the forward modeling technique for geological stratum calibration and the stacking velocity time-depth conversion were proved to be very effective in improving the accuracy of seismic survey.
\end{abstract}

Keywords: Acquisition geometry, forward modeling, geological stratum calibration, less explored region.

\section{INTRODUCTION}

3D seismic survey is an effective method for prospecting geological structure such as fold, fault, collapsed column, goaf and coal seam erosion zone. But in the west part of China, due to the complicated geological condition, borehole data are rarely found and the prospecting level is very low. In the hope of speeding up the coalmine construction, a survey region started 3D seismic survey just after the stage of pre-survey, or merely in the stage of theoretical analysis of small coal mine mining situation. The lack of survey data brings difficulties for 3D seismic acquisition geometry design, reflection wave geological stratum calibration and time-depth conversion, and affects the accuracy of the prospecting result [1].

\section{THE DETERMINATION OF 3D SEISMIC SURVEY PARAMETERS}

3D seismic survey parameters include spatial sample interval, maximum offset, and maximum vertical distance, fold times and so on $[2,3]$.

\subsection{Binning Size}

In data acquisition system, only the binning size that satisfies the formula can have complete spatial sample frequency [4].

$b \leq \frac{V_{\text {int }}}{4 F_{\text {max }} \sin \theta}$

Where $\mathrm{b}$ denotes the binning size, Vint denotes the upper layer velocity, Fmax is the highest non aliasing frequency and $\theta$ is the dip angle [4].

\footnotetext{
*Address correspondence to this author at CCTEG Xi'an Research Institute, Xi'an City, Shaaxi Province, 710077, P.R. China; Tel: +86 2981778129 ; Fax:+8629 81778129; E-mail: thw801025@126.com
}

1874-1495/15

\subsection{Determination of Maximum Offset}

The proper maximum offset depends on these factors: NMO distortion rate, accuracy error of velocity analysis and reflection coefficient stability.

NMO procedure can distort waveform, especially for large offset. So when designing spread length, the NMO distortion of effective reflection waves from shallow and medium layer should be taken into account; the distortion should be restricted to a certain range. The relation between NMO distortion rate and spread length is:

$k=\sqrt{1+\frac{x^{2}}{t_{0}^{2} v^{2}}}-1$

Where $\mathrm{k}$ denotes the distortion rate, $\mathrm{x}$ denotes the offset, $\mathrm{t} 0$ is the two-way travel time and $\mathrm{v}$ is the stacking velocity.

When designing spread length, this distortion effect should be taken into consideration and be restricted in less than $10 \%$, so that the signal frequency is less damaged.

The RMS velocity and stacking velocity are computed based on the normal moveout, since the normal moveout increases along with the offset; the maximum offset must be large enough to guarantee high velocity accuracy [5]. The maximum offset should meet the accuracy requirement of the velocity analysis.

$x \geq \sqrt{\frac{2 \mathrm{t} 0}{\mathrm{f}\left[(\mathrm{v}-\Delta \mathrm{v})^{-2}-\mathrm{v}^{-2}\right]}}$

Here $\Delta V$ is the velocity error, $\mathrm{x}$ is the spread length, $\mathrm{v}$ is the stacking velocity, $\mathrm{f}$ is the main frequency of reflection wave and t0 is the two-way travel time of target stratum [6].

When the incidence angle approaches the critical angle, the reflection coefficient changes drastically, therefore to keep a relatively stable reflection coefficient, the incidence angle should be smaller than the critical angle (generally, less than $40^{\circ}$, in high dip angle area, less than $30^{\circ}$ ), so maximum offset limitation can be calculated as: 
$x \leq 2 * \sum_{\mathrm{i}=1}^{\mathrm{n}} \mathrm{h}_{\mathrm{i}} \tan \theta_{\mathrm{i} 0}$

Where hi is the thickness of layer i, $\theta \mathrm{i} 0$ is the critical angle of layer $i$ and $x$ is the maximum offset.

\subsection{Determination of Max Vertical Distance}

Max vertical distance is the maximum vertical offset between survey line and shot point in an active acquisition geometry [6].

$y_{\max } \leq \frac{v}{\sin \psi} \sqrt{2 t_{0} \delta t}$

Here, ? denotes the maximum dip angle (degree), ? is the maximum time difference (s) within one gathered CMP, often being a quarter of the apparent period of effective reflection wave. $\mathrm{V}$ is the average velocity $(\mathrm{m} / \mathrm{s}), ?$ is the two-way travel time (s) of target stratum. (AUTHOR: The variables are missing here)

\subsection{Fold Times}

Fold times is determined by experience or experiment [7].

All the acquisition parameters above are determined by drilling data or mining data, so in a survey region whichlacks these data, these parameters must be estimated by some other method.

\section{3D SEISMIC SURVEY EXAMPLE IN JIXIN COALMINE}

Jixin coalmine is located in Jimusar, Xinjiang province, and is composed of three local small coalmines: Hongshanwa coalmine, Anxin coalmine, Shichanggoujixin coalmine. Before this 3D seismic survey, no drilling data has been found, nor geological survey data about this region and the geology structure are unknown. The outcrop and mining data show that the strata of this survey region are composed of lower sub-formation of Badaowan Formation of lower Jurassic and the Quaternary system. The coal seams are in Badaowan Formation of lower Jurassic, and among them, enable seams are mainly $7,6,4,3$ coal seams. 7 coal seam is about $3 \sim 6 \mathrm{~m}$ thick, 6 coal seam is about $10 \sim 18 \mathrm{~m}, 4$ coal seam is about $1.5 \sim 5 \mathrm{~m}$ and the 3 coal seam is about $1.9 \sim 3 \mathrm{~m}$. The distance between floor of seam 7 and roof of seam 6 is about 10 25 m, and the distance between floor of seam 6 and roof of seam 4 is about $25 \mathrm{~m}$, floor of seam 4 and roof of seam 3 are about $1 \mathrm{~m}$. The bury depth of coal seam varies between $100 \sim 450 \mathrm{~m}$, and the dip angle is about $25^{\circ}$.

\subsection{Preliminary Design of Acquisition Parameters}

Based on the 3D seismic survey experience of adjacent area and acquisition parameter argument [8], 8-line-8-shot geometry is chosen, in which inline interval is $40 \mathrm{~m}$, trace interval is $10 \mathrm{~m}, \mathrm{CDP}$ binning is $5 \mathrm{~m} \times 10 \mathrm{~m}$, active trace number in a single survey line is 48 , fold times is 24 , shooting off end, the maximum offset is $514 \mathrm{~m}$, the maximum vertical distance is $210 \mathrm{~m}$, and the minimum offset for shallow part is $175 \mathrm{~m}$, while for deeper part it is $194 \mathrm{~m}$.

\subsection{D Experiment}

Using the explosive parameters of single point experiment, a 2D survey line experiment was carried out using preliminary designed acquisition parameters. Survey line is designed along the direction of dip, and active receivers are 48 , trace interval is $10 \mathrm{~m}$, shooting off end with zero offset and fold time is 24 . The experiment data is processed and the result section is shown in Fig. (1).

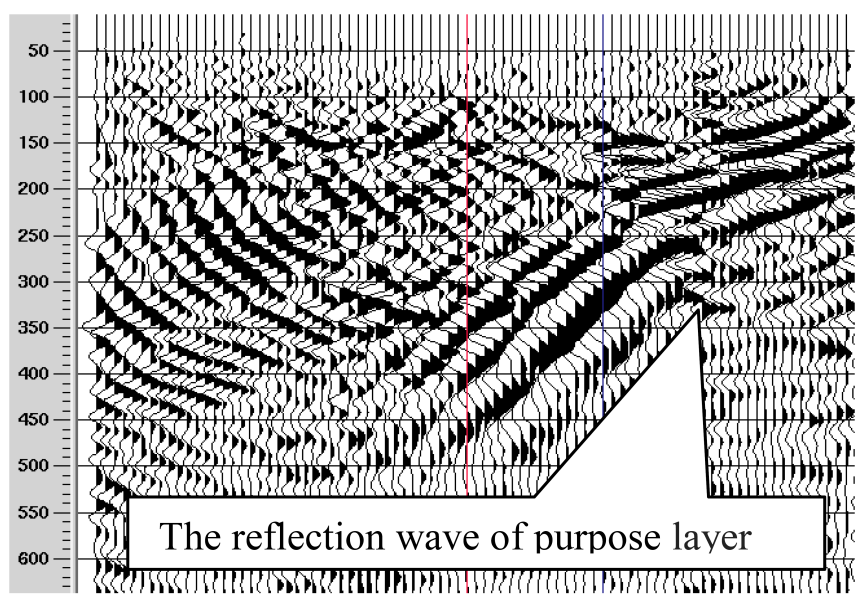

Fig. (1). Data of experimental survey line after stack.

From Fig. (1), it can be seen that data with fold times of 24 has a good $\mathrm{S} / \mathrm{N}$ ratio, and meets the prospection need. The travel time of reflection wave from target layer varies between $0.2 \mathrm{~s} \sim 0.5 \mathrm{~s}$, the stack velocity of target layer at the shallow part is $3100 \mathrm{~m} / \mathrm{s}$, and that of at the deep part is $3300 \mathrm{~m} / \mathrm{s}$, with the highest valid frequency being $120 \mathrm{~Hz}$. The dip angle of shallow part is $15^{\circ}$, and that of the deep part is $45^{\circ}$.

\subsection{Re-argument of Acquisition Parameters}

Putting RMS velocity, highest valid frequency of reflection wave and dip angle of target layer into equation (1), it can be computed that the CDP binning size should be smaller than $9.724 \mathrm{~m} \times 9.724 \mathrm{~m}$ to ensure that space aliasing does not occur. Putting RMS velocity, two-way travel time of reflection wave and dip angle of target layer into equation (2), it can be seen that the maximum offset should be less than $720 \mathrm{~m}$. Putting the depth and dip angle of target layer into equation (3), it can be seen that the maximum offset should be less than $600 \mathrm{~m}$. Taking these two results into consideration, $650 \mathrm{~m}$ is chosen as the maximum offset. From equation (5), it can be computed that the maximum vertical distance should be less than $250 \mathrm{~m}$. Compared with the preliminary design, it can be seen that the CDP binning size does not meet the requirement of eliminating space aliasing, and the CDP binning size should be adjusted to $5 \mathrm{~m} \times 5 \mathrm{~m}$. The maximum offset and maximum vertical distance of preliminary design meet the requirement, but the minimum offset of shallow part is too large, which can cause blank in shallow seismic data, while the minimum offset of deep part is too small, which can cause blank in deep seismic data, and both lead to sharp shrinkage of effective control area.

The final acquisition parameters are adjusted to: 8-line-5shot acquisition geometry with inline interval of $20 \mathrm{~m}$, trace interval of $10 \mathrm{~m}$, and CDP binning size is $5 \mathrm{~m} \times 5 \mathrm{~m}$. Active receive traces in a single line are 48, fold times is 24 , shooting off end, maximum offset is $502 \mathrm{~m}$, maximum 
vertical distance is $150 \mathrm{~m}$. The minimum offset in shallow part is $60 \mathrm{~m}$, the minimum offset in deep part is $620 \mathrm{~m}$.

\subsection{Data processing Quality Evaluation}

After data process of the whole survey area, the $\mathrm{S} / \mathrm{N}$ ratio is relatively high, the energy of reflection wave from target layer is very strong, and is easy to be recognized and traced. The geology structure is quite clear (Fig. 2).

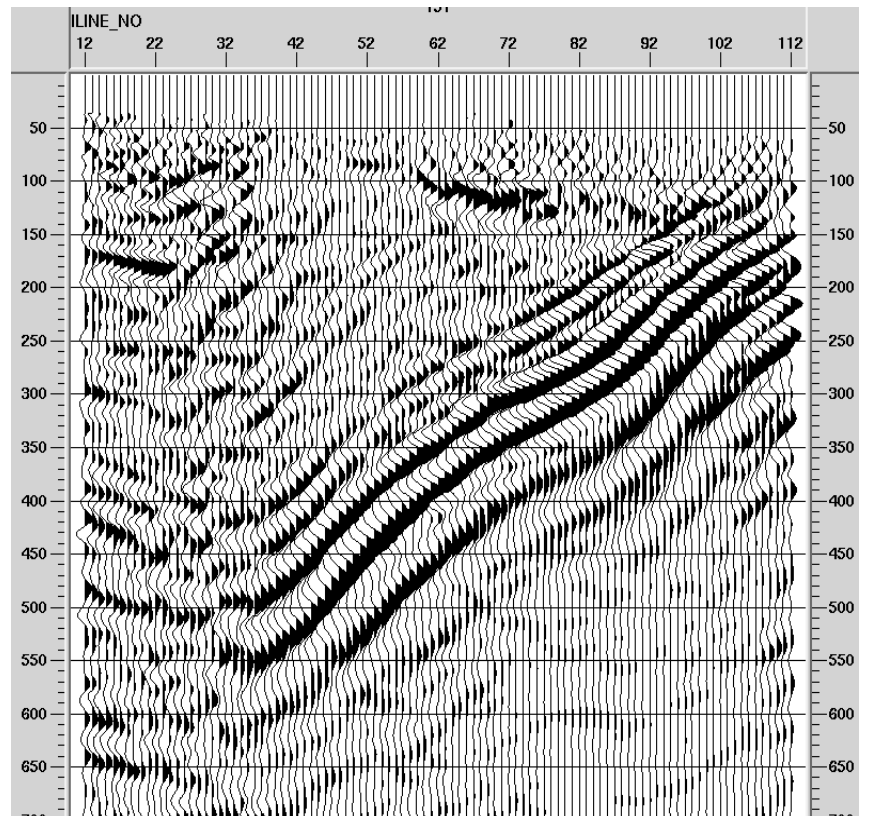

Fig. (2). Final migration sections.

\subsection{Seismic Data Fine Interpretation}

There are three steps in 3D seismic data interpretation: geological stratum calibration by reflection wave, geology structure interpretation and time-depth conversion.

\subsubsection{Geological Stratum Calibration}

Common stratum calibration methods include VSP bridge connection, adjacent pilot stratum, forward modeling and time-depth conversion. But due to lack of borehole data, the above four methods cannot work. So a new method must be found. Constructing a geological model (Fig. 3) by outcropping data and mining data, forward modeling is conducted, and the result (Fig. 4) can be used to calibrate the reflection wave to a certain stratum. Fig. (5) is the standard time profile of survey area, and by comparing Fig. (4) with Fig (5), it is found that the calibration is reasonable. By considering the similarity of waveform, phase continuity, amplitude and frequency characteristic, and by comparing the relation between multi wave groups, the reflection wave is traced. For some reflection wave that cannot be traced in the whole survey area, it is traced and compared locally.

\subsubsection{Preliminary Time-depth Conversion}

The conventional time-depth conversion equation is $\mathrm{H}=1 / 2 \times \mathrm{V} \times \mathrm{T} 0$; this method needs borehole data or mining data to calibrate seismic velocities to establish seismic velocity field of the whole survey area (requiring an evenly distributed velocity samples). By using this method, timedepth conversion was carried out and the result is shown in Table 1.

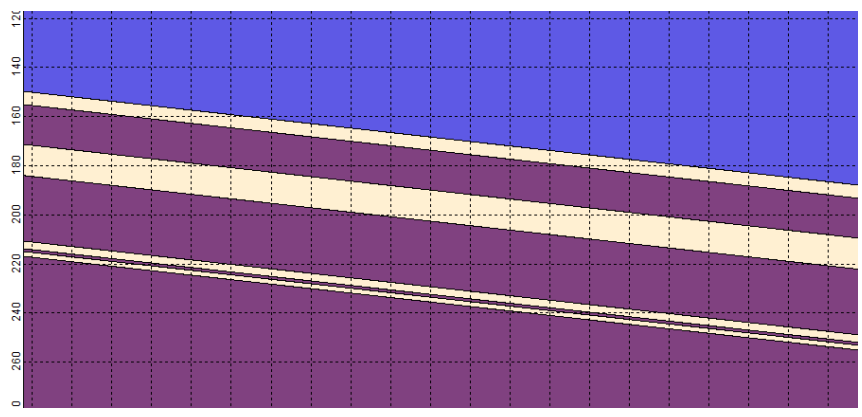

Fig. (3). A simple geology model of survey area.

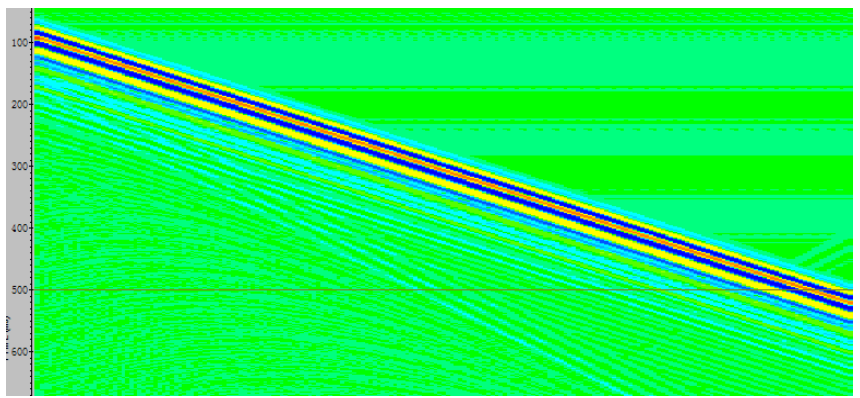

Fig. (4). Synthetic data of geology model.

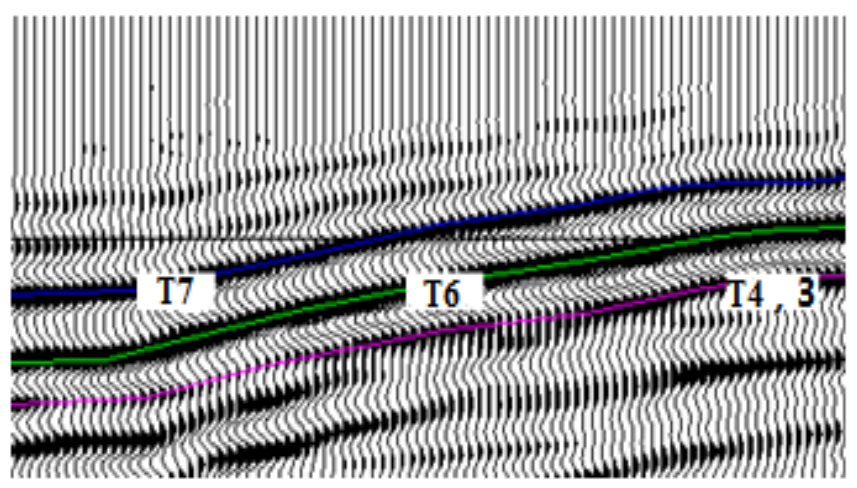

Fig. (5). Standard time section of survey area.

In data processing, velocity analysis was carried out using $100 \mathrm{~m} \times 100 \mathrm{~m}$ grid, which equals to a drilling network of $100 \mathrm{~m} \times 100 \mathrm{~m}$. Based on this assumption, stack velocity was converted into interval velocity, and the velocity field was constructed along the target coal seam layer. Then the interval velocity and average velocity were obtained from outcrops in coal seam roadway. Dividing average velocity by interval velocity, a velocity coefficient $\mathrm{k}$ can be obtained, and the contour map of $\mathrm{k}$ can be drawn. Multiplying $\mathrm{k}$ contour map with the interval velocity contour, an average velocity contour can be obtained, and the travel time of reflection wave can be used from coal seam in the stack section, computing a coal seam floor contour map. Using this coal seam floor contour map, it can be observed that the coal seam floor elevation of 6th coal seam was well predicted in the 4 explorations (Table 1). 
Table 1. Predicted elevation of $6^{\text {th }}$ coal mine floor.

\begin{tabular}{|c|c|c|c|c|}
\hline Type & Borehole A1 & Borehole A2 & Borehole A3 & Borehole A4 \\
\hline Conventional convert method & 385 & 390 & 420 & 370 \\
\hline Stack velocity conversion method & 410 & 444 & 470 & 400 \\
\hline Actual elevation by borehole data & 415 & 425 & & 410 \\
\hline
\end{tabular}

Two months later after the structural concept, borehole A1, A2 and A4 were completed. From Table 1, it can be seen that the coal seam floor elevation predictions of two methods are different, and the stack velocity conversion method is more accurate.

\subsubsection{Final Time-depth Conversion}

Adding drilling data of borehole A1, A2, A4 to stack velocity time-depth conversion, and after adjusting $\mathrm{k}$ value contour, a new velocity contour can be obtained. Conducting stack velocity time-depth conversion again, it can be predicted that the depth of A3 borehole is $485 \mathrm{~m}$.

\subsection{Verification of Survey Result}

Drilling data of borehole A3 show that the elevation of floor of 6th coal seam is $489 \mathrm{~m}$, and the roadway mining data shows that the elevation errors of coal seam floor are below $1.5 \%$. Two faults of both $7 \mathrm{~m}$ fault throw are verified by roadway mining, and the horizontal error of fault position is no more than $10 \mathrm{~m}$.

\section{CONCLUSION}

CDP binning size is the fundamental parameter in data acquisition geometry design as it decides the shot interval and receiver interval and affects the total cost. The size of CDP binning should meet the need of eliminating space aliasing. The re-argument of data acquisition parameters can ensure obtaining good quality seismic data. In a survey area lacking borehole data, forward modeling technique can be used to determine which stratum the reflection wave comes from. Time-depth conversion using stack velocity can improve the accuracy of coal seam floor elevation determination.

\section{CONFLICT OF INTEREST}

The authors confirm that this article content has no conflict of interest.

\section{ACKNOWLEDGEMENTS}

Declared none.

\section{REFERENCES}

[1] A. M. Zhang, Z. Y. Ding, and Z. Luo, "Application and development of 3D seismic technology in green mining," Journal of China University of Mining \& Technology, vol. 33, no. 2, pp. 229-232, 2004 (In Chinese).

[2] Y. W. Liu, and C. Xiu, "Three parameter velocity analysis of converted wave and NMO method," Oil Geophysics Prospecting, vol. 40, no.5, pp. 504-509, 2005.

[3] Y. Z. Xu, R. F. Cui, and D. M. Pan, "Application of seismic inversion based on model for igneous distribution in coalfield," Journal of China University of Mining \& Technology, vol. 17, no. 1, pp. 11-14, 2007, (In Chinese).

[4] X. 1. Gong, and J. D. Lin, "Study on time-depth transform method of three-dimensional seismic information interpretation in coal field," Coal Geology of China, vol. 14, no. 2, pp. 65-68, 2002.

[5] L. Y. Jia, N. T. Lin, and L. Zhao, "Analysis on the methods for time-depth conversion of seismic wave," Sci-thch Information Development \& Economy, vol. 21, no. 9, pp. 193-197, 2011.

[6] Y. Z. Xu, "Comparison between several seismic inversion methods and their application in mountainous coal fields of western China", Mining Science and Technology (China), vol. 20, no.4, pp. 585590,2010

[7] J. Wang, "Problems in the time-depth conversion of 3D data and their solution," Geophysical Prospecting for Petroleum, vol. 34, no. 2, pp. 110-115, 1995.

[8] C. Yin, and J. X. Xu, "attributes analysis and optimum design of 3Dgeometry," Oil Geophysics Prospecting, vol. 40, no. 5, pp. 495498, 2005.

(C) Hongwei and Wenxin; Licensee Bentham Open.

This is an open access article licensed under the terms of the Creative Commons Attribution Non-Commercial License (http://creativecommons.org/licenses/ by-nc/4.0/) which permits unrestricted, non-commercial use, distribution and reproduction in any medium, provided the work is properly cited. 